University of Nebraska - Lincoln

DigitalCommons@University of Nebraska - Lincoln

$4-1-2002$

\title{
Dissociative electron attachment to molecules in the gas phase and in rare gas solids
}

\author{
Paul Burrow \\ University of Nebraska - Lincoln, pburrow1@unl.edu \\ Kayvan Aflatooni \\ University of Nebraska-Lincoln, kaflatoo@fhsu.edu
}

Follow this and additional works at: https://digitalcommons.unl.edu/physicsburrow

Part of the Physics Commons

Burrow, Paul and Aflatooni, Kayvan, "Dissociative electron attachment to molecules in the gas phase and in rare gas solids" (2002). Paul Burrow Publications. 7.

https://digitalcommons.unl.edu/physicsburrow/7

This Article is brought to you for free and open access by the Research Papers in Physics and Astronomy at DigitalCommons@University of Nebraska - Lincoln. It has been accepted for inclusion in Paul Burrow Publications by an authorized administrator of DigitalCommons@University of Nebraska - Lincoln. 


\title{
Dissociative electron attachment to molecules in the gas phase and in rare gas solids
}

\author{
P. D. Burrow ${ }^{\text {a) }}$ and K. Aflatooni $i^{\text {b) }}$ \\ Department of Physics and Astronomy, University of Nebraska-Lincoln, Lincoln, Nebraska 68588-0111
}

(Received 18 December 2001; accepted 16 January 2002)

\begin{abstract}
Measurements of dissociative electron attachment (DEA) cross sections in chloroalkanes and chlorofluoromethanes have shown strong correlations between the peak DEA cross sections and the vertical attachment energies (VAEs) of these compounds. We explore the extent to which these gas phase data can be used to predict such cross sections for molecules embedded within or on the surface of a Kr solid. Effective VAEs are computed that include polarization of the solid by the anion and effects due to electron motion in the lattice. Comparisons are made with recent surface and bulk measurements and show good agreement for $\mathrm{CF}_{3} \mathrm{Cl}$ both within and on the surface. Satisfactory agreement is found for $\mathrm{CH}_{3} \mathrm{Cl}$ in the bulk but not on the surface. (C) 2002 American Institute of Physics. [DOI: 10.1063/1.1458536]
\end{abstract}

\section{INTRODUCTION}

The calculation of cross sections for the dissociative electron attachment (DEA) process in the gas phase, $e$ $+A B \rightarrow A B^{-*} \rightarrow A+B^{-}$, remains a significant challenge to theorists because of the extreme sensitivity of the process to properties of the transient negative ion state $A B^{-*}$, particularly its energy and lifetime. Additional complexity is added when this reaction occurs in molecules on surfaces or in solids. The conceptually simplest such environment may well be with target molecules embedded in a rare gas lattice. In two tour de force experiments, Nagesha and Sanche ${ }^{1}$ and Fabrikant et $\mathrm{al}^{2}{ }^{2}$ reported absolute cross sections for production of stable negative ions in $\mathrm{CF}_{3} \mathrm{Cl}$ and $\mathrm{CH}_{3} \mathrm{Cl}$, respectively, within a solid lattice of krypton. In more recent work, ${ }^{3}$ these authors have reported new and more reliable values of the absolute cross sections for these two species both within and on the surface of the rare gas film. The measurements were accompanied ${ }^{2,3}$ by theoretical DEA cross sections obtained by using a semiempirical $R$-matrix method ${ }^{4}$ extended to incorporate the effects of the condensed matter environment. In brief, these additions took account of the polarization interaction between the negative ion and the medium and effects related to the effective mass of the electron moving in the lattice. Two models were put forth. The first utilized only a polarization interaction and was most successful for molecules on the surface of the rare gas solid. The second incorporated both polarization and effective mass considerations and was applied to molecules within the solid.

In the present report, we examine the extent to which DEA cross sections for such compounds in and on the rare gas solid can be obtained from recently determined empirical relationships between gas phase cross sections and the vertical attachment energies (VAEs) required to form the temporary negative ion states in the ground state geometries of

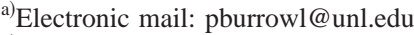

${ }^{b}$ Present address: Department of Physics, Fort Hays State University, Hays, KS 67601; electronic mail: kaflatoo@fhsu.edu
}

the neutral molecules. ${ }^{5-7}$ Parallel to the theoretical treatment by Nagesha et al., ${ }^{3}$ we incorporate effects due to polarization and the effective mass of the electron in an empirical manner using literature values for these quantities.

\section{BACKGROUND}

In work reported elsewhere, ${ }^{5-7}$ the DEA cross sections and VAEs of a large number of mono- and polychloroalkanes and a small set of fluorinated chloromethanes have been measured. Each of these compounds has one or more lowlying unoccupied $\mathrm{C}-\mathrm{Cl} \sigma^{*}$ molecular orbitals, and the attachment of a free electron into the lowest of these [the lowest unoccupied molecular orbital (LUMO)] forms a transient negative ion state that is the dominant contributor to the DEA process at low electron energies. VAEs were determined by electron transmission spectroscopy (ETS), ${ }^{8}$ and the values span a range from approximately 0.4 to $3.45 \mathrm{eV}$. Total DEA cross sections were measured as described elsewhere. ${ }^{5,7}$ At these low energies, the dominant negative ion produced is $\mathrm{Cl}^{-}$.

The purpose of the previous work was to determine whether the peak DEA cross sections in these compounds could be correlated with the VAEs. The primary outcome is summarized in Fig. 1, in which are plotted the peak values of the DEA cross sections as a function of VAE for the chloroalkanes (closed symbols) and fluorochloromethanes (open symbols). A clear correlation exists for each family. The solid and dashed lines indicate fits to the data. For the chloroalkanes, the dashed line is given by

$$
\sigma_{\mathrm{DEA}}^{\text {peak }}=5.41 \times 10^{-\left(16+0.613 \mathrm{VAE}^{2.01}\right)} \mathrm{cm}^{2}
$$

and was determined by fitting to measurements in 32 chloroalkanes covering the range of VAEs from 0.6 to $2.7 \mathrm{eV}$. The average deviation of the data from the line is $38 \%$, excluding the apparently anomalous point for $\mathrm{CH}_{2} \mathrm{Cl}_{2}$.

The result for $\mathrm{CH}_{3} \mathrm{Cl}$ at a measured VAE of $3.45 \mathrm{eV}$ warrants a few comments. Because of the small magnitude of its DEA cross section and problems with trace amounts of 


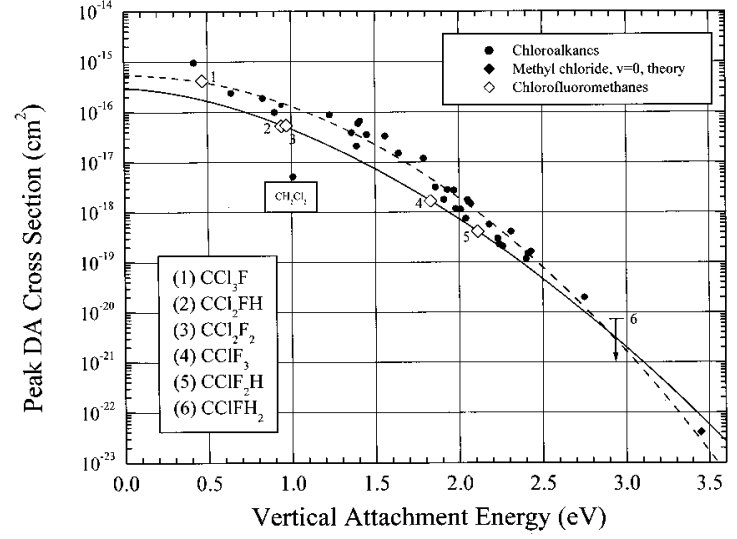

FIG. 1. Peak cross sections for the dissociative electron attachment process as a function of vertical attachment energy (VAE) in the chloroalkanes (closed symbols) and chlorofluoromethanes (open symbols). The dashed and solid lines indicate best fits to these two data sets, respectively. The anomalous point for $\mathrm{CH}_{2} \mathrm{Cl}_{2}$ is not included in the fit. The $\mathrm{CH}_{3} \mathrm{Cl}$ data point was computed from theory (Refs 4 and 10).

contaminants, only an upper bound to the peak DEA cross section at room temperature could be determined. ${ }^{9}$ However, Fabrikant ${ }^{4,10}$ has computed a value of $4 \times 10^{-23} \mathrm{~cm}^{2}$ for DEA from the vibrational ground state of $\mathrm{CH}_{3} \mathrm{Cl}$, using his semiempirical $R$-matrix approach. This point is indicated in Fig. 1 as a closed diamond, but was not included in the fit to the experimental data. We note, however, that it is in quite good agreement with that predicted by extension of the bestfit line to higher VAE.

The solid line in Fig. 1 for the chlorofluoromethanes is expressed by

$$
\sigma_{\text {DEA }}^{\text {peak }}=2.97 \times 10^{-\left(16+0.81 \mathrm{VAE}^{1.69}\right)} \mathrm{cm}^{2}
$$

and shows the best fit to results in four fluorinated chloromethanes with VAEs ranging from 0.9 to $2.2 \mathrm{eV}$. The deviations from this line are quite small, but this reflects in part the small number of data points.

The correlations between the peak DEA cross sections and VAE are discussed in more detail elsewhere. ${ }^{5,7}$ In brief, we have argued that they arise primarily from the monotonic dependences of the temporary negative ion lifetimes on VAE. Our ETS data ${ }^{6}$ show that the widths, $\Gamma$, of these resonances vary with VAE in a manner consistent with the Wigner threshold law ${ }^{11}$ for electron tunneling through an angular momentum barrier. This is illustrated for the chloroalkanes in Fig. 2 in which is plotted a quantity that is proportional to the width of the resonance structure in the total scattering cross section $^{6}$ as a function of VAE. The solid line shows a best fit to the data having a threshold-law form, $\Gamma \propto \mathrm{VAE}^{l+1 / 2}$, where $l$ is the angular momentum quantum number characterizing the barrier. After making allowance for smaller contributions from Franck-Condon overlaps, as discussed in more detail elsewhere, ${ }^{5}$ the resonance widths are found to vary as $\mathrm{VAE}^{\approx 1.5}$, consistent with electron tunneling through an $l$ $=1$ barrier. This behavior could be anticipated from the $p_{\sigma}$ character of the $\mathrm{C}-\mathrm{Cl} \sigma^{*}$ orbital.

The results suggest a picture in which the angular momentum barrier responsible for trapping the electron into the

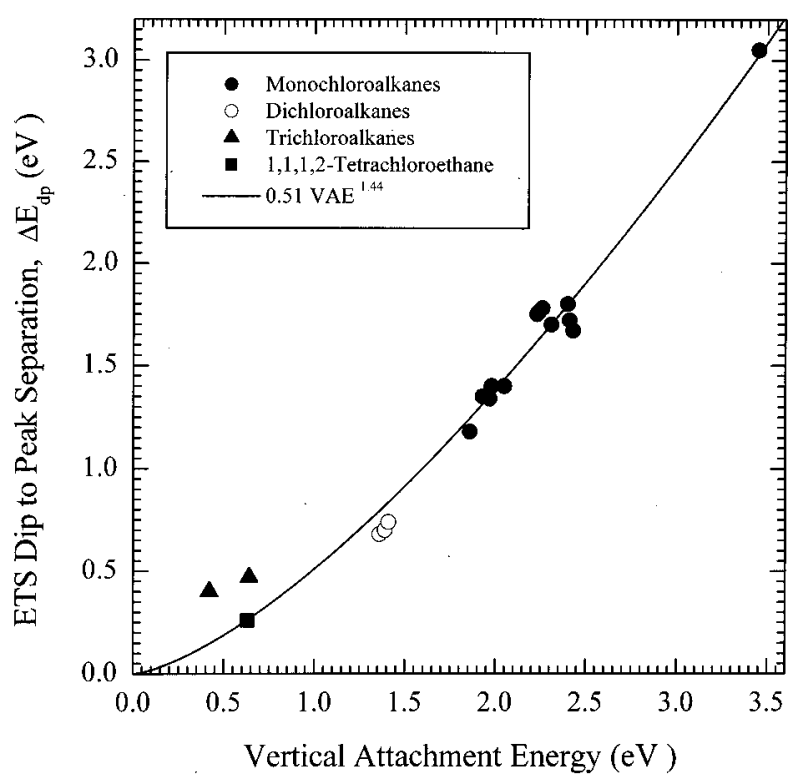

FIG. 2. The widths of resonance peaks in the total scattering cross section as measured by the dip to peak energy separation in the derivative of transmitted electron current as a function of the VAE of various chloroalkanes. The solid line shows a best fit to these data of the form $A \times \mathrm{VAE}^{n}$.

LUMO, and through which it must tunnel to escape, is essentially the same for all the compounds of a given family. Changing the energy of the temporary negative ion, that is, VAE, simply shifts its location higher or lower inside this barrier thus shortening or lengthening the lifetime. As shown by O'Malley, ${ }^{12}$ the DEA cross section is exponentially sensitive to the latter. The cross section also depends exponentially on the time required for the components of the negative ion to separate to the crossing with the neutral potential curve. However, based on simple modeling of the potential curves, the variation of separation times with VAE was found to be weaker. ${ }^{6}$ As Eq. (1) shows, VAE enters to the power of 2.01 in the best-fit curve. Our treatment suggests that approximately 1.5 of this exponent arises from the dependence on lifetime and the remaining amount to the variation of separation times with VAE.

Finally, it is important to note that the actual electron energies at which the cross sections reach their maximum values are not involved in these correlations. Only the VAEs are employed.

\section{DISCUSSION}

The striking relationships between the peak DEA cross section and VAE in the two molecular families illustrated in Fig. 1 span almost seven orders of magnitude in cross section. In the present paper we suggest a different way to utilize these data. Rather than considering the data as a collection of discrete points for different molecules, we propose viewing the best-fit line as the "trajectory" that would be followed by any one particular compound under circumstances that alter its VAE. For example, placing a molecule on a polarizable medium will stabilize the negative ion state relative to the neutral molecule by the associated surface 
polarization energy, $E_{p}^{s}$, thus lowering the VAE from its gas phase value. This yields an effective $\mathrm{VAE}_{\text {eff }}=\mathrm{VAE}-E_{p}^{s}$ that can be used in Eq. (1) or (2) to predict the associated peak DEA cross section. This procedure thus corresponds to the first model utilized by Nagesha et $a l .^{3}$ but with the arduous $R$-matrix scattering calculation replaced with the empirical relationships illustrated in Fig. 1.

For the case of molecules placed within the rare gas lattice, Nagesha $e t a l .^{3}$ incorporated a second model that included a stabilization owing to bulk polarization and a more interesting effect associated with the Bloch wave description of a free electron moving in the lattice. Because the effective mass of the electron in this medium is less than that in vacuum, the electron momentum in the medium, for a given kinetic energy, is less than that in the gas phase, leading to a reduction of the decay width. We can connect this immediately to our results in Fig. 2, which show resonance widths as a function of VAE. Again regarding the solid best-fit line as the "trajectory" followed by the width of a particular compound under circumstances in which the VAE is changed, we see that a reduction of the width is equivalent to a decrease in effective VAE. From Fig. 1, it is clear that such a reduction produces a larger peak DEA cross section.

We incorporate this effect heuristically with the following discussion. Consider an autodetaching electron tunneling through the angular momentum barrier and emerging into either the rare gas lattice or the vacuum with a kinetic energy $E$. We have then that

$$
E=\frac{\hbar^{2} k^{2}}{2 m}=\frac{\hbar^{2} k^{* 2}}{2 m^{*}},
$$

where the symbols with asterisks indicate values within the rare gas lattice. If the reduced mass, $m^{*}$, is less than $m$, then $k^{*}<k$. From the Wigner threshold law for tunneling through a spherical barrier, we have that $\Gamma \propto k^{2 l+1}$. If $k^{*}<k$, then $\Gamma^{*}<\Gamma$, as noted by Nagesha et $_{\text {al. }}{ }^{3}$

For a given electron energy $E$, Eq. (3) implies that

$$
\left(\frac{k^{*}}{k}\right)^{2}=\frac{m^{*}}{m} \text {. }
$$

Thus we can incorporate the threshold law in terms of the ratio of the two resonance widths as

$$
\frac{\Gamma^{*}}{\Gamma}=\left[\left(\frac{k^{*}}{k}\right)^{2}\right]^{l+1 / 2}=\left(\frac{m^{*}}{m}\right)^{l+1 / 2} .
$$

At this point, we invoke our empirically determined relationship, shown in Fig. 2, connecting $\Gamma$ and VAE, namely that $\Gamma \propto(\mathrm{VAE})^{l+1 / 2}$. We associate the width in the medium, $\Gamma^{*}$, with an effective VAE that we label $\mathrm{VAE}_{\text {eff }}$ and write

$$
\frac{\Gamma^{*}}{\Gamma}=\left(\frac{m^{*}}{m}\right)^{l+1 / 2}=\left(\frac{\mathrm{VAE}_{\mathrm{eff}}}{\mathrm{VAE}}\right)^{l+1 / 2} .
$$

Thus the reduced mass of the electron in the medium leads to an effective $\mathrm{VAE}_{\text {eff }}$ given by $\mathrm{VAE}_{\text {eff }}=(m * / m) \mathrm{VAE}$ in the absence of other effects. Bearing in mind the role of polarization in the medium described earlier, we can summarize the inclusion of both these effects, corresponding to the second model of Nagesha et al., ${ }^{3}$ with an effective VAE given by

$$
\mathrm{VAE}_{\text {eff }}=(m * / m)\left(\mathrm{VAE}_{\mathrm{gas}}-E_{p}^{b}\right),
$$

where $E_{p}^{b}$ corresponds to the bulk polarization energy. As mentioned earlier, when the molecule is on the surface, $\mathrm{VAE}_{\text {eff }}=\mathrm{VAE}_{\mathrm{gas}}-E_{p}^{s}$, and $E_{p}^{s}$ is the surface polarization energy.

\section{RESULTS}

In this section, we examine how well these effective VAEs predict the measured surface and bulk DEA cross sections when used with Eq. (1) or (2). First, we require experimental values for $E_{p}^{s}, E_{p}^{b}$, and $m^{*}$ in Kr. By observing shifts from the gas phase energy of the $\mathrm{N}_{2}^{-}$temporary anion state when $\mathrm{N}_{2}$ resides on the surface or in bulk $\mathrm{Kr}$, Michaud and Sanche ${ }^{13}$ arrive at values of $E_{p}^{s}=0.72 \mathrm{eV}$ and $E_{p}^{b}=1.15 \mathrm{eV}$. These values are also used in the analysis in Nagesha et al. $^{3}$

Determination of the electron effective mass in $\mathrm{Kr}$ is more problematic. Nagesha et al. ${ }^{3}$ employ $m^{*} / m=0.42$ from Ref. 14. A case can also be made for a somewhat higher value. A detailed experimental study of $m^{*}$ in an argon solid by Perluzzo et al. ${ }^{15}$ found $m^{*} / m=0.53 \pm 0.01$. Somewhat earlier, the same workers, Bader et al. ${ }^{16}$ found that $m_{\mathrm{Kr}} * / m_{\mathrm{Ar}}{ }^{*}=1.18$. Using the value for argon by Perluzzo et al. ${ }^{15}$ in this ratio yields $m_{\mathrm{Kr}}{ }^{*} / m=0.63$.

Table I summarizes the present predictions for the peak DEA cross sections of $\mathrm{CF}_{3} \mathrm{Cl}$ and $\mathrm{CH}_{3} \mathrm{Cl}$ inside the $\mathrm{Kr}$ medium ("bulk" values) or on the surface, together with the measured values from Nagesha $e \mathrm{all}^{3}$ and the semiempirical $R$-matrix calculations of this same reference. We associate error limits of $\pm 38 \%$ on our predicted values arising from the average scatter in the chloroalkane data shown in Fig. 1. Nagesha et al. ${ }^{3}$ report experimental errors of $\pm 48 \%$ in the measured values. Predicted peak cross sections in the bulk are shown for $m^{*} / m=0.42$ as well as 0.63 . The $R$-matrix calculations $^{3}$ were carried out only with 0.42 .

\section{A. $\mathrm{CF}_{3} \mathrm{Cl}$ inside the $\mathrm{Kr}$ medium}

The predicted peak cross sections with the two choices of $m^{*} / m$ straddle the experimental result, with both in agreement well within the experimental errors. The predicted cross section using $m^{*} / m=0.63$ falls in slightly better agreement. The $R$-matrix calculation does not fare as well here, lying a factor of 3.3 higher than the experimental cross section.

\section{B. $\mathrm{CF}_{3} \mathrm{Cl}$ on the $\mathrm{Kr}$ surface}

The peak cross sections in this environment are all in excellent agreement, again well within experimental error. Nagesha et al. ${ }^{3}$ also report $R$-matrix calculations using $E_{p}$ values of 1.15 and $1.25 \mathrm{eV}$ as well as $0.72 \mathrm{eV}$. Our predicted peak cross sections using these parameters also track these results closely.

\section{C. $\mathrm{CH}_{3} \mathrm{Cl}$ inside the $\mathrm{Kr}$ medium}

Similar to the case with $\mathrm{CF}_{3} \mathrm{Cl}$ in the bulk, the predicted peak cross sections straddle the experimental value with the two choices of $m^{*} / m$. In this example, however, with $m^{*} / m=0.42$ the cross section lies within the respective error 
TABLE I. Peak cross sections for electron attachment in (bulk) and on (surface) $\mathrm{Kr}$ solid.

\begin{tabular}{|c|c|c|c|}
\hline $\mathrm{CF}_{3} \mathrm{Cl}$ & Present prediction & Experiment $^{\mathrm{a}}$ & $R$-matrix Calc. ${ }^{\mathrm{b}}$ \\
\hline Bulk $(m * / m=0.63)$ & $1.90 \times 10^{-16} \mathrm{~cm}^{2}$ & $2.1 \times 10^{-16} \mathrm{~cm}^{2}$ & $\cdots$ \\
\hline Bulk $\left(m^{*} / m=0.42\right)$ & $2.37 \times 10^{-16} \mathrm{~cm}^{2}$ & & $6.9 \times 10^{-16} \mathrm{~cm}^{2}$ \\
\hline \multirow[t]{3}{*}{ Surface } & $\begin{array}{l}3.2 \times 10^{-17} \mathrm{~cm}^{2} \\
\left(E_{p}=0.72 \mathrm{eV}\right)\end{array}$ & $4.3 \times 10^{-17} \mathrm{~cm}^{2}$ & $\begin{array}{l}4.0 \times 10^{-17} \mathrm{~cm}^{2} \\
\left(E_{p}=0.72 \mathrm{eV}\right)\end{array}$ \\
\hline & $\begin{array}{l}1.12 \times 10^{-16} \mathrm{~cm}^{2} \\
\left(E_{p}=1.15 \mathrm{eV}\right)\end{array}$ & & $\begin{array}{l}1.05 \times 10^{-16} \mathrm{~cm}^{2} \\
\left(E_{p}=1.15 \mathrm{eV}\right)\end{array}$ \\
\hline & $\begin{array}{l}1.41 \times 10^{-16} \mathrm{~cm}^{2} \\
\left(E_{p}=1.25 \mathrm{eV}\right)\end{array}$ & & $\begin{array}{l}1.95 \times 10^{-16} \mathrm{~cm}^{2} \\
\left(E_{p}=1.25 \mathrm{eV}\right)\end{array}$ \\
\hline \multicolumn{4}{|l|}{$\mathrm{CH}_{3} \mathrm{Cl}$} \\
\hline Bulk $(m * / m=0.63)$ & $2.8 \times 10^{-17} \mathrm{~cm}^{2}$ & $\sim 1 \times 10^{-16} \mathrm{~cm}^{2}$ & $\cdots$ \\
\hline Bulk $\left(m^{*} / m=0.42\right)$ & $1.45 \times 10^{-16} \mathrm{~cm}^{2}$ & & $\begin{array}{l}4.75 \times 10^{-17} \mathrm{~cm}^{2} \\
\text { (Mod anion curve) }\end{array}$ \\
\hline \multirow[t]{2}{*}{ Surface } & $1.3 \times 10^{-20} \mathrm{~cm}^{2}$ & $1.2 \times 10^{-17} \mathrm{~cm}^{2}$ & $\begin{array}{l}\sim 2 \times 10^{-19} \mathrm{~cm}^{2} \\
\text { (Orig anion curve) }\end{array}$ \\
\hline & & & $\begin{array}{l}\sim 1 \times 10^{-17} \mathrm{~cm}^{2} \\
\text { (Mod anion curve) }\end{array}$ \\
\hline
\end{tabular}

${ }^{\text {a}}$ Reference 3.

${ }^{\mathrm{b}}$ Using $m * / m=0.42$.

limits while that for $m * / m=0.63$ is approximately a factor of 3 smaller than experiment. The $R$-matrix value is smaller by a factor of 2 . The latter cross section, however, was reached after modification of the negative ion potential curve from that originally calculated for gas phase studies. ${ }^{4}$ The motivations for this are discussed in Nagesha et al. ${ }^{3}$

\section{D. $\mathrm{CH}_{3} \mathrm{Cl}$ on the $\mathrm{Kr}$ surface}

For this environment, the predicted peak cross section is almost three orders of magnitude smaller than the measured value. The original $R$-matrix treatment of this process ${ }^{2}$ similarly yielded a cross section that was about 50 times too small. Nagesha et al. ${ }^{3}$ note that agreement with experiment can be reproduced only with substantial semiempirical adjustments. This, in part, was a consideration in modifying the negative ion potential curve as mentioned previously. By decreasing the $\mathrm{C}-\mathrm{Cl}$ distance at which the anion and neutral curves cross, a cross section in much better agreement with the experimental value could be attained.

Given the data in Fig. 1, the only parameter available in the present analysis is the surface polarizability. To reach the measured value of the peak cross section, $E_{p}^{s}$ would have to be $1.8 \mathrm{eV}$ rather than the value of $0.72 \mathrm{eV}$ used in our model and which gave a good result in $\mathrm{CF}_{3} \mathrm{Cl}$. We can only provide one possible rationale for this, namely that the static dipole moment of $\mathrm{CH}_{3} \mathrm{Cl}$ is substantially larger than that of $\mathrm{CF}_{3} \mathrm{Cl}$, and thus that the $E_{p}^{s}$ inferred from $\mathrm{N}_{2}^{-}$on the $\mathrm{Kr}$ surface ${ }^{13}$ may not be appropriate.

\section{CONCLUSIONS}

Gas phase DEA cross sections and VAEs within a family of closely related molecules can be used to estimate the peak DEA cross sections of these molecules when they are placed within a Kr lattice, given the bulk polarization energy and the effective mass of the free electron moving in the lattice. A similar treatment incorporating only the surface polarization energy is successful for $\mathrm{CF}_{3} \mathrm{Cl}$ but fails for $\mathrm{CH}_{3} \mathrm{Cl}$. This result could imply that the $\mathrm{CH}_{3} \mathrm{Cl}$ anion potential curve on the surface and in the bulk is altered from that found in the gas phase, as suggested by Nagesha et al. ${ }^{3}$ and consequently that the connection with the gas phase data we describe here might not be useful. Alternatively, since modification of the $\mathrm{CF}_{3} \mathrm{Cl}$ curve was not required, the problem may lie with the surface polarization energy of $\mathrm{CH}_{3} \mathrm{Cl}^{-}$being substantially greater than that found for $\mathrm{N}_{2}^{-}$owing to the large dipole moment of $\mathrm{CH}_{3} \mathrm{Cl}$. Additional measurements with molecules in these families with differing dipole moments would be useful to resolve this puzzle. Measurements in media with a range of different values for the electron effective mass would also test the suitability of the present empirical approach.

Finally, we note that the gas phase DEA cross sections discussed here are those with peaks at electron energies generally well above $\approx 0.1 \mathrm{eV}$. The DEA process also yields a small narrow peak at nominally zero energy that becomes more pronounced as VAE decreases. ${ }^{5}$ At very low VAEs $(<0.3 \mathrm{eV})$, this component may also contribute substantially to the total cross sections, however, our treatment does not include it.

\section{ACKNOWLEDGMENTS}

This work was supported by the National Science Foundation. We are pleased to acknowledge many useful discussions with our colleagues Gordon Gallup and Ilya Fabrikant.

\footnotetext{
${ }^{1}$ K. Nagesha and L. Sanche, Phys. Rev. Lett. 78, 4725 (1997).

${ }^{2}$ I. I. Fabrikant, K. Nagesha, R. Wilde, and L. Sanche, Phys. Rev. B 56, R5725 (1997).
} 
${ }^{3}$ K. Nagesha, I. I. Fabrikant, and L. Sanche, J. Chem. Phys. 114, 4934 (2001).

${ }^{4}$ I. I. Fabrikant, J. Phys. B 27, 4325 (1994).

${ }^{5}$ K. Aflatooni and P. D. Burrow, J. Chem. Phys. 113, 1455 (2000); D. M. Pearl and P. D. Burrow, ibid. 101, 2940 (1994).

${ }^{6}$ K. Aflatooni, G. A. Gallup, and P. D. Burrow, J. Phys. Chem. A 104, 7359 (2000).

${ }^{7}$ K. Aflatooni and P. D. Burrow, Int. J. Mass. Spectrom. 205, 149 (2001).

${ }^{8}$ L. Sanche and G. J. Schulz, Phys. Rev. A 5, 1672 (1972).

${ }^{9}$ S. C. Chu and P. D. Burrow, Chem. Phys. Lett. 172, 17 (1990).

${ }^{10}$ I. I. Fabrikant (private communication).
${ }^{11}$ E. P. Wigner, Phys. Rev. 73, 1002 (1948).

${ }^{12}$ T. F. O’Malley, Phys. Rev. 150, 14 (1966).

${ }^{13}$ M. Michaud and L. Sanche, J. Electron Spectrosc. Relat. Phenom 51, 237 (1990).

${ }^{14}$ Rare Gas Solids, edited by M. L. Klein and J. A. Venables (Academic, London, 1976) Vols. 1 and 2, p. 522.

${ }^{15}$ G. Perluzzo, G. Bader, L. G. Caron, and L. Sanche, Phys. Rev. Lett. 55, 545 (1985).

${ }^{16}$ G. Bader, G. Perluzzo, L. G. Caron, and L. Sanche, Phys. Rev. B 30, 78 (1984). 